\title{
A Fast and Robust Method of Focusing
}

\section{Xu Dijian ${ }^{1, a}, Z$ hu Hongjun ${ }^{2, b}$, Shi Jinliang ${ }^{3, c}$, Chen Guorong ${ }^{4, d}$}

\section{${ }^{1}$ Metallurgical Performance Detection and Equipment Engineering Technology Research Center, ChongQing University of Science \& Technology, China}

axdj1003@163.com, ${ }^{b}$ redreda@qq.com, ${ }^{c}$ Shijinliang@tom.com, ${ }^{d}$ cqcrg@sina.com

\section{Keywords:Image preocessing, Canny algorithm, Autofocus}

\begin{abstract}
In the measuring technology of microscopy, in order to realize high focusing accuracy and also strong noise suppression, the author approves a new focusing method for microscopy on the base of principle of microscope imaging and focus. The method uses Canny algorithm to recognize the edge of the measured object. When the edge of the measured object is the narrowest, the microscope gets the best focusing. The experiment shows that compared with the classical focusing evaluation function, this method is characteristic of unimodality, accuracy, sensibility and noise immunity. The method is an autofucusing method with combination property, and it is especially suitable for high precision and speed microscopic measurement.
\end{abstract}

\section{Introduction}

With the development of modern manufacturing industry and detecting technique, the tool microscope is widely used as the detecting instrument and is becoming automatic and intelligent. Microscopy measurement is concerned about image acquire, feature extraction, target identification, auto focus and noise suppression. Among these, the autofocusing is one of the key techniques. So the focusing quality can effect the position, definition of the image[1] and the accuracy of measurement. At present, there are many focusing appraisal functions, such as VAR, EIG, ELI, and so on. But all kinds of focusing algorithm have different focusing accuracy, sensitivity and repeatability for the different images. And most algorithms are based on infinitesimal calculus and use the high frequency component to appraise the off-focus, so it is sensitive to noise. The acquiring image of the tool microscope is sketch of the measured objects and the outline is line. The aim of automatic focusing is to get the clear measured edge not all the edge. But the edge is easy to be falsely detected due to noise. So this paper proposes the Automatic focus evaluation function suitable to tool microscope with high antinoise ability, which we call BW method.

\section{Principle of focusing}

In the system of microscope and imaging, when the focusing is correct, one point light source $\mathrm{A}(\mathrm{x}, \mathrm{y})$ of the object plane is imaged as one image point in the image plane. While when the focusing is off, one point light source of the object plane is imaged as one facula in the image plane.

Any optical image system can be thought as the ideal Gauss imaging system. One image edge can be considered as the superposition of images of countless point source near the edge. For simplicity, we discuss the focusing principle on the base the line edge vertical with the lord. When the focusing is correct, the image of the line edge is also a line, while the focusing is off, the image of the line edge is not clear and looks like a band. In order to describe the points in this area, we should construct one 2-demension coordinate with the edge direction as $\mathrm{y}$, and vertical to the edge as $\mathrm{x}$. in the microscope, the images from the acquiring system have the almost same gray value in the same $\mathrm{y}$ with the approximately $\frac{\partial f(x, y)}{\partial y}=0$. But the gray value of off-focus image is related to $\mathrm{x}$ in the space. The image points can be expressed as:

$$
f\left(x-x_{0} ; b, c, \sigma\right)=(b+c U(x)) \otimes g(x ; \sigma)=b+\frac{c}{2}\left(1-\operatorname{erf}\left(\frac{x-x_{0}}{\sigma \sqrt{2}}\right)\right)
$$


Here, $U(x)$ is unit step function and $g(x ; \sigma)$ is Gauss function. When the scale of off-focusing is greater, $\sigma$ is greater. The gray value of the edge is showed in figure 1.

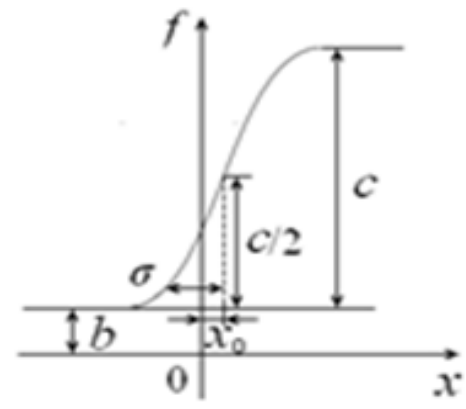

Fig1 The edge modle

Form figure 1, we can easily see that when the focus is correct, the image of the edge approaches the step edge and the gray gradient of the edge is the bigger with the least width. While when focus is off, the edge of image is slope type with less gray gradient and bigger width.

\section{Focus evaluation function and its measurement}

Focus evaluation function.In micro-measurement, the accuracy is the first factor and the width of the image edge is smaller and the measurement is more precise. So, the basic standard of evaluating focus is the width of the image edge. The focus evaluation function with correct focusing and the narrowest image edge is the best function.

Let the maximum of the measured edge width be Bmax, the measured image width $\mathrm{B}$, when the focusing is more clear, width of edge is narrower shown in figure 1. and the difference between $\mathrm{B}$ and Bmax is the biggest. So we conclude that the best evaluating focus function is

$$
F=B_{\text {max }}-B
$$

When $\mathrm{F}$ is bigger, the focus is clearer. When $\mathrm{F}$ is the biggest, the focus is complete. The edge width can be measured by the numbers of the edge pixel with complex computation. We know that the number of the pixels vary with the width of the edge. When the edge is narrowest, the number is least. So the form (2) can be changed as

$$
F=N E_{\max }-N E
$$

Here NE is number of pixel in the measured edge and NEmax is the maximum.

Confirm the edge poing. The traditional edge detecting algorithm include Roberts operator, Prewitt operator, Sobel operator(First order differential operator) and Laplacian operator、 LOG operator(second order differential operator). Usually these operators use first derivative maximum points or second order derivative zero as the candidate edge point. And set the suitable thresholds and extract image edge. This method is simple, easy to realize and quick to compute. But infinitesimal calculus is sensitive to noise, so it is easy to be disturbed. So it is limited in practical use. In 1986, Canny proposed the best criterion to edge detecting, that is MSIR, positioning precision standards, and single edge response criterion, and then he deduced the best edge detecting algorithm. This method first use Gauss filter to smooth the image and use the window to compute the largest value and direction of the gray gradient, and then restrain the non-largest for the gradient graph, and apply double thresholds to detect and combine the edge from the candidate edge points.

However, Canny detector is sensitive to its parameter. For this reason, the input parameters were selected automatically and objectively using a method based on statistical correspondence which presented by Yitzhaky and Peli [9]. We would establish ground truth according to the method proposed in [9]. Then the best parameter set can be determined according to the Chi-square criterion.

\section{Experimental Verification}

The standard of focusing evaluation function is accuracy, unimodality, sensitivity, repeatability 
and practical applicity. This standard can be used for the rough focusing and fine focusing with different strict degree.

In order to verify the performance of BW, we do many experiments, including rough focusing, fine focusing and noise. We choose regular objects and use different methods VAR, EIG and ELI to compare with BW. In experiment, BW use 30 pixels as the focusing window, and the others use the hole image and the window.

Normalization.In order to compare the different functions on accuracy, unimodality, sensitivity, repeatability and practical application, we must put all the results normalization. Aiming at not changing accuracy, unimodality, sensitivity, repeatability and practical application, the position between the extreme value point and inflexion point and object distance must be invariable. So we choose the linear function to realize normalization, list in (4).

$$
F_{T}=\left(F-F_{\min }\right) /\left(F_{\max }-F_{\min }\right)
$$

Rough focusing. Rough focusing is the process of quickly determining the potential focus area using unimodality, in which fine focus is unnecessary. In this experiment, we mainly test the unimodality and repeatability.

(1)unimodality

when the focal length varies in the step of $50 \mu \mathrm{m}$ in the range of $1.5 \mathrm{~mm}$ near focusing clear area, the normalization results of focus evaluation function is listed in figure 2.

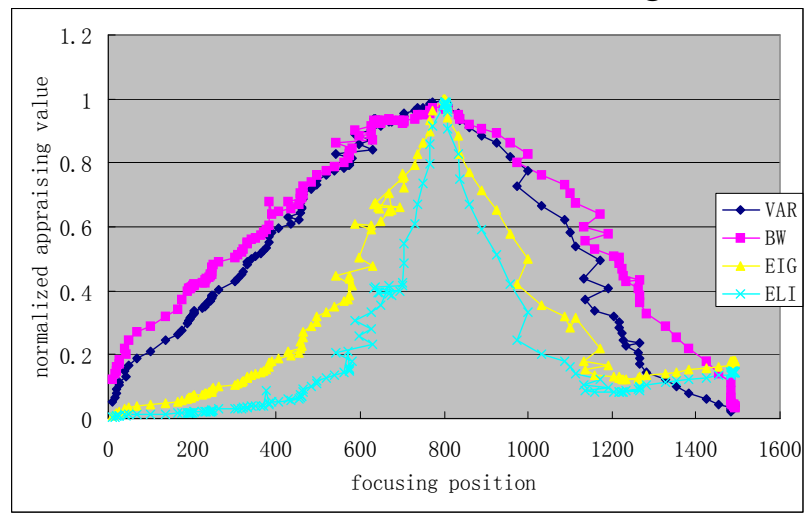

Fig.2. Focus curves

From the figure 2, we can know that BW and VAR have a consistent convexity-concavity in the hole range without local maximum and good unimodality. This feature can be used to confirm the direction of step motor.

(2)repeatability

in the many repeat focusings, the method with the least mean-square deviation is the best algorithm. We use different methods to focus the same object in the step of 50mm for 30 times. We find there exists little difference.

Fine focusing.Fine focusing requires the focus function is accurate and precise and has high sensitivity. In this experiment, we mainly test accuracy, unimodality, sensitivity, repeatability.

(1)unimodality

when the focal length varies in the step of $1 \mu \mathrm{m}$ in the range of $170 \mu \mathrm{m}$ near focusing clear area, the normalization results of accuracy, unimodality, sensitivity are listed in figure 3.

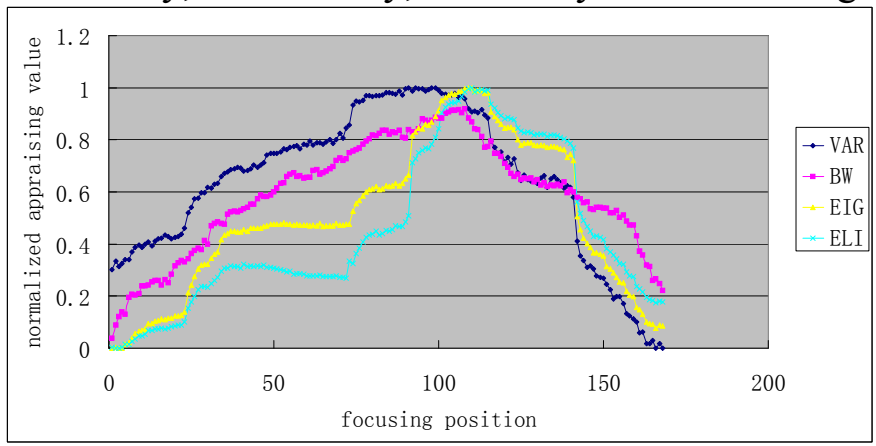

Fig.3. Focus curves II 
From the figure 3, we can know that BW, EIG and ELI have no local maximum and good unimodality. The unimodality of VAR is relatively bad.

(2) Accuracy

The best focusing position computed by EIG and ELI are different from BW, which shows there exists difference between these methods. That is to say, in EIG and ELI, when the function value is the biggest, but the edge width is not the narrowest. While the edge width is the narrowest computed on the base of VAR and BW.

(3) Sensitivity

From figure 3, we know that the sensitivity of EIG, ELI is the best, then the BW, last VAR.

(4) Repeatability.

We use different methods to focus the same object in the step of $1 \mu \mathrm{m}$ for 50 times. We find that there exists difference listed in table 1.

Tab.1 The test results of focusing functions on fine auto-focusing stage

\begin{tabular}{lllll}
\hline $\begin{array}{l}\text { Criterion } \\
\text { function }\end{array}$ & WB & VAR & EIG & ELI \\
\hline $\begin{array}{l}\text { Mean } \\
\text { value } / \mathrm{mm}\end{array}$ & 12.8595 & 12.8638 & 12.8726 & 12.8787 \\
$\begin{array}{l}\text { Standard } \\
\text { deviation/mm }\end{array}$ & 0.0008 & 0.0021 & 0.0011 & 0.0012 \\
\hline
\end{tabular}

Anti-noise experiment.We changed the focal length in the step of $1 \mu \mathrm{m}$ in the range of $500 \mu \mathrm{m}$ near focusing clear area and then induced the Gauss noise and salt-and-pepper noise to the intake of image to test the ability of anti-noise. We focus the same object for 30 times. The standard deviation of focusing functions in noise suppression is listed in table 2.

Tab.2 The standard deviation of focusing functions in noise suppression

\begin{tabular}{lllll}
\hline $\begin{array}{l}\text { Criterion } \\
\text { function }\end{array}$ & WB & VAR & EIG & ELI \\
\hline $\begin{array}{l}\text { Gauss } \\
\text { noise/mm }\end{array}$ & 0.0121 & 0.0963 & 0.0525 & 0.0413 \\
$\begin{array}{l}\text { salt-and-pepp } \\
\text { er noise } / \mathrm{mm}\end{array}$ & 0.0048 & 0.0837 & 0.0512 & 0.0322 \\
\hline
\end{tabular}

\section{Conclusion}

This paper proposes a new focusing method for micro measurement. From the above experiment results, we can know that this algorithm has high anti-noise ability with high sensitivity. And in the large range, there is no local maximum with good unimodality. All these will simplify the searching difficulty in the process of automatic focusing and narrow the range of searching, only to improve the focusing speed. On the other hand, this algorithm can reduce the complexity.

\section{Acknowledgements}

This research is supported by Chongqing Science and Technology Research Projects (No.cstc2011pt-gc70007, cstc2012gg-yyjs0118), the Achievements support Foundation of Chongqing Municipal Education Commission (No. kjzh11221, kj121413, 201028), national natural sciences fund of China(Project NO. 51275547, and Research Foundation of Chongqing University of Science \& Technology (No. CK2010Z10, CK2011B01).

\section{References}

[1] E.P. Krotkov. Focusing[J]. International Journal of Computer Vision. 1987,1:223 237.

[2]Murali Subbarao, Jenn-Kwei Tyan. Selecting. the optimal focus measure for autofocusing and depth-from-focus[J]. IEEE Transactions on Pattern Analysis and Machine Intelligence, August 1998, 20(8):864 870. 
[3]Lighart G, Groen F. A comparison of different autofocus algorithms[C]. IEEE 6th International Conference on Pattern Recognition, 1982. pp. 597 600.

[4]Mike Heath, Sudeep Sarkar, Thomas Sanocki, Kevin Bowyer. Comparison of edge detectors: a methodology and initial study[C]. IEEE Transactions on Computer Vision and Pattern Recognition, Jun 1996. pp.143 148 .

[5]Zhang Le, Jiang Wei. Application of multi-resolution analysis in anti-noise capability of auto-focusing algorithm[J]. Acta Optica Sinica, 2007, 27(12):2150 2154

[6]Tihanyi E. Z., Barron J. L..Spatio-temporal edge focusing[J]. IEEE Transactions Proceedings of the 10th International Conference on Pattern Recognition. Jun 1990, 1:213 216

[7] Haralick R. Digital step edges from zero crossing of second directional derivatives[J].IEEE Trans on Pattern Analysis and Machine Intelligence, 1984,6(1):58-68.

[8]Canny J. A computational approach to edge detection[J].IEEE Transactions on Pattern Analysis and Machine Intelligence, 1986,8(6):679-698.

[9]Y. Yitzhaky, E. Peli, A method for objective edge detection evaluation and detector parameter selection, IEEE Transactions on Pattern Analysis and Machine Intelligence 25 (8) (2003) 1027-1033.

[10]Frans C.A. Groen, Ian T. Young, Guido Ligthart. A comparison of different focus functions for use in autofocus algorithms[J]. Cytometry, 1985, 6:81 $\sim 91$.

[11] Kang-Sun Choi, Sung-Jae KO. New autofocusing technique using the frequency selective weighted median filter for video cameras[J]. IEEE Transactions on Consumer Electronics, August 1999, 3(45): 820 827. 